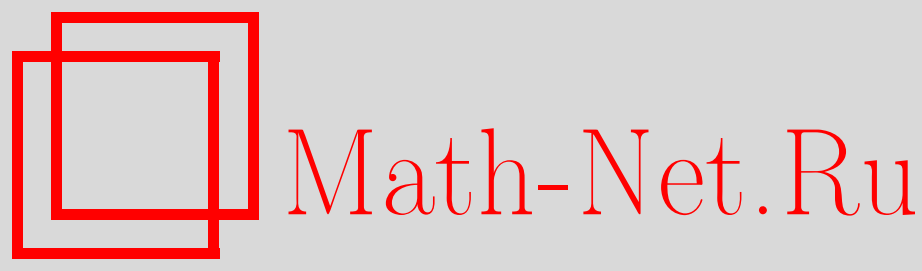

С. А. Гриценко, Е. А. Карацуба, М. А. Королёв, И. С. Резвякова, Д. И. Толев, М. Е. Чанга, Научные достижения Анатолия Алексеевича Карацубы, Совр. пробл. матем., 2012, выпуск 16, 7-30

DOI: https://doi.org/10.4213/spm30

Использование Общероссийского математического портала Math-Net.Ru подразумевает, что вы прочитали и согласны с пользовательским соглашением http://www.mathnet.ru/rus/agreement

Параметры загрузки:

IP : 52.205 .19 .152

26 апреля 2023 г., 12:23:42

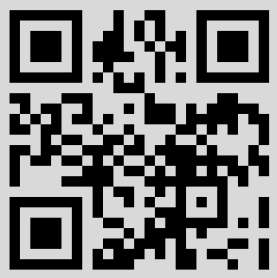




\title{
Научные достижения Анатолия Алексеевича Карацубы
}

\author{
С. А. Гриценко, Е. А. Карацуба, М. А. Королёв, И. С. Резвякова, Д. И. Толев, \\ M. Е. Чанга
}

\section{1. Научная биография}

Анатолий Алексеевич Карацуба родился 31 января 1937 г. в г. Грозном. В 1944-1954 гг. учился в средней мужской школе № 6 г. Грозного и окончил ее с серебряной медалью. Уже в младших классах Толя Карацуба проявил исключительные математические способности, решая задачи математического кружка для старшеклассников.

В 1954 г. Анатолий Карацуба поступил на механико-математический факультет МГУ им. М. В. Ломоносова. Среди особенно любимых лекторов он отмечал Н. Г. Четаева, П. С. Александрова, Л.С. Понтрягина, а наиболее любимыми преподавателями, ведущими семинары, были Л. А. Скорняков и А. Ф. Филиппов.

C 1-го курса А.А. Карацуба слушал специальные курсы и принимал участие в работе научных семинаров: на 1-м курсе - семинаров П.С. Александрова и П.К. Рашевского (по дифференциальной геометрии); на 1-м и 2-м курсах - Е. Б. Дынкина (по алгебре, анализу и теории вероятностей); на 2-м курсе - семинаров А. Н. Колмогорова, П. С. Александрова, Р. М. Джабар-заде (по ЭВМ); на 3-м курсе - А.Н. Колмогорова, Н. М. Коробова; на 4-м курсе - А. Н. Колмогорова, Н. М. Коробова, В. М. Успенского, А. О. Гельфонда; на 5-м курсе Н. М. Коробова, А. О. Гельфонда.

Первым руководителем научных работ А. А. Карацубы был А. Н. Колмогоров, под руководством которого он защитил курсовую работу 3-го курса под названием "Вариации функций двух переменных" и курсовую работу 4-го курса "Эксперименты с автоматами". Основные результаты последней работы в виде статьи "Решение одной задачи из теории конечных автоматов" (см. [1]) были переданы в 1958 г. для публикации в журнал «Успехи математических наук». В этой работе А. А. Карацубой было найдено неулучшаемое значение для длины кратчайшего эксперимента, позволяющего привести так называемый автомат Мура в известное состояние (при условии, что любые два состояния этого автомата различимы), и это явилось первым из важнейших вкладов А. А. Карацубы в математику, в такую ее область, как кибернетика (информатика).

Решение другой поставленной А. Н. Колмогоровым задачи о сложности умножения привело к изобретению А. А. Карацубой первого быстрого метода вычислений (см. [2]), названного впоследствии умножением Карацубы, и послужило созданию нового направления вычислительной математики - теории быстрых алгоритмов. Быстрое умножение является не только фундаментальным теоретическим результатом, но и одним из самых широко внедренных в ЭВМ (как в software, так и в hardware) математических методов. С одной стороны, быстрый метод умножения Карацубы опровергал гипотезу Колмогорова о том, что обычное умножение в столбик является асимптотически наибыстрейшим способом умножения. С другой стороны, внедрение быстрого умножения и других построенных на этой идее Карацубы алгоритмов позволило значительно повысить эффективность работы ЭВМ, недаром в журнале «Pour la Science» за 2000 г. (см. [3]) умножение Карацубы названо “одним из самых полезных результатов в математике".

В настоящее время идея Карацубы, на которой основано его быстрое умножение, используется во многих алгоритмах и фигурирует в литературе под названиями Divide and Conquer, Binary Splitting и др. Однако появление в 1960 г. быстрого умножения, опровергающего гипотезу А.Н. Колмогорова, явилось для него столь большой неожиданностью, что он закрыл 
свой семинар по кибернетике. Это определило выбор дальнейшего пути в математике для Анатолия Карацубы, который сосредоточился на занятиях теорией чисел.

После окончания в 1959 г. механико-математического факультета МГУ А. А. Карацуба поступил в аспирантуру к Н.М̈. Коробову. В 1962 г. он защитил кандидатскую диссертацию "Рациональные тригонометрические суммы специального вида и их приложения" и начал работать на механико-математическом факультете МГУ. В 1966 г. А. А. Карацуба защитил докторскую диссертацию "Метод тригонометрических сумм и теоремы о среднем" и стал научным сотрудником Математического института им. В. А. Стеклова АН СССР (МИАН). C этого времени и до конца жизни он был сначала научным сотрудником, а затем заведующим отделом теории чисел МИАН и по совместительству на полставки профессором механико-математического факультета МГУ.

В МГУ А. А. Карацуба вел два научных семинара и читал специальные курсы по теории чисел. Его ученики и коллеги отмечают, что лекции он читал замечательно. Ему удавалось теоретико-числовые теоремы, иногда исключительно сложные, делать ясными для слушателей.

А. А. Карацуба создал свою научную школу. Среди его непосредственных учеников - пятнадцать кандидатов наук (Григорий Колесник, Сергей Воронин, Геннадий Архипов, Владимир Чубариков, Михаил Петечук, Владимир Плаксин, Зарулло Рахмонов, Ильгар Джаббаров, Алла Лаврик, Сергей Гриценко, Гавхар Негматова, Дойчин Толев, Максим Королев, Марис Чанга, Ирина Резвякова), семеро из которых впоследствии стали докторами наук.

В теорию чисел А. А. Карацуба внес настолько значительный вклад, что в полном объеме его идеями, изобретениями и методами будут пользоваться не только его современники, но и многие будущие поколения математиков. Среди основных результатов А. А. Карацубы можно назвать: построение $p$-адического метода в теории тригонометрических сумм, что привело к новым границам $L$-рядов Дирихле и решению проблемы распределения дробных долей многочлена с целыми коэффициентами по модулю, равному степени простого числа, к новому p-адическому доказательству теоремы о среднем И. М. Виноградова, занимающей центральное место в методе тригонометрических сумм Виноградова, а также к решению проблемы Хуа Ло-кена о показателе сходимости особого интеграла проблемы Терри; создание нового метода решения тернарных мультипликативных задач; создание теории кратных тригонометрических сумм Г. Вейля; получение оценки функции Харди в проблеме Варинга; получение принципиально нового результата по проблеме Артина о p-адическом представлении нуля формой произвольной степени; создание нового метода оценок коротких сумм Клоостермана. В теории дзета-функции Римана к основным результатам А. А. Карацубы принадлежат: доказательство гипотезы Сельберга о количестве нулей дзета-функции Римана $\zeta(s)$ на критической прямой; доказательство аналога гипотезы Сельберга о распределении нулей $\zeta(s)$ на почти всех "коротких" промежутках критической прямой; построение нового метода исследования нулей дзета-функции Римана на почти всех "сверхкоротких" промежутках критической прямой; создание нового метода исследования нулей функций (таких, например, как функция Дэвенпорта-Хэйльбронна), представимых в виде линейных комбинаций $L$-рядов Дирихле; получение принципиально новых результатов в многомерной проблеме делителей Дирихле; доказательство нижних оценок максимума модуля дзета-функции в малых областях критической полосы и на малых промежутках критической прямой; создание метода исследования аргумента дзета-функции Римана $\zeta(s)$ на "коротких" промежутках критической прямой. В теории характеров Дирихле к основным результатам А. А. Карацубы относятся: создание нового метода оценок коротких сумм характеров Дирихле в конечных полях, оценок линейных сумм характеров по сдвинутым простым числам, оценок сумм характеров от многочленов с простым аргументом, а также сумм характеров на аддитивных последовательностях и сумм значений характеров Дирихле "с весами". В последние годы помимо теоретико-числовых исследований А.А. Карацуба занимался также применением построенных им в теории чисел методов в задачах квантовой физики. 
Основные исследования А. А. Карацубы опубликованы более чем в 160 научных статьях (см. [4]) и в монографиях [5]-[9].

В статье "О математических работах профессора Карацубы" (см. [10]), фрагменты которой используются в дальнейшем тексте, ученики Анатолия Алексеевича Г. И. Архипов и В. Н. Чубариков так описывают особенности научных работ А. А. Карацубы: "При изложении трудов замечательных ученых естественно выделить какие-нибудь характерные и яркие черты их творчества. Такими отличительными чертами в научной деятельности профессора Карацубы являются комбинаторная изобретательность, основательность и определенная законченность результатов."

Анатолий Алексеевич Карацуба умер 28 сентября 2008 г. после несвоевременной и нерезультативной операции. Внезапность его болезни и смерти не позволили завершить многие начатые им исследования.

А. А. Карацуба был, пожалуй, одним из самых преданных математике ученых нашего времени, внесшим вклад, сравнимый с вкладом классиков науки прошлого, результаты которых напрямую влияли на развитие науки и даже повседневной жизни. Он никогда не занимался политической и общественной деятельностью, не состоял членом никаких партий. Он любил математику. До последних дней жизни думал над решением проблем. Его результаты доказывают - математика тоже его любила.

\section{2. Первые работы. Информатика}

Далее в тексте статьи будут использоваться следующие обозначения: $\pi(N)$ - количество простых чисел, не превосходящих $N ; \tau(N)$ - количество делителей числа $N$; $i$ мнимая единица, $i^{2}=-1 ; C, C_{1}, \ldots, c, c_{1}, \ldots$ - положительные абсолютные (если специально не оговаривается другое) постоянные, в разных утверждениях, вообе говоря, разные; при $B>0$, $B \rightarrow+\infty$ или $B \rightarrow 0$ запись $1 \ll A / B \ll 1$ означает, что существуют константы $C_{1}>0$ и $C_{2}>0$ такие, что $C_{1} \leqslant|A| / B \leqslant C_{2}$; для вещественного $\alpha$ запись $\|\alpha\|$ означает расстояние до ближайшего целого, $\|\alpha\|=\min (\{\alpha\}, 1-\{\alpha\})$, где $\{\alpha\}-$ дробная часть $\alpha ; \varepsilon>0$ - произвольное сколь угодно малое фиксированное число; постоянные в знаках $O$ являются абсолютными (если специально не оговаривается другое).

2.1. Автоматы. В статье Эдварда Мура "Умозрительные эксперименты на последовательных машинах" (см. [11]) $S$-( $n ; m ; p)$-машина (или автомат) определяется как имеющее $n$ состояний, $m$ входных символов и $p$ выходных символов устройство. Позднее такие $S$-машины стали называть автоматами Мура. В конце статьи, где доказывается девять теорем о структуре $S$ и экспериментах с $S$, в главе "Новые проблемы" Мур формулирует задачу об улучшении оценок полученных им в двух последних теоремах.

Теорема (Мур; см. [11, теорема 8]). Пусть задана произвольная $S$ - $(n ; m ; p)$-машина такая, что каждые два ее состояния отличимы одно от другого. Тогда существует эксперимент длины $n(n-1) / 2$, который устанавливает (находит) состояние $S$ в конце этого эксперимента.

В 1957 г. А.А. Карацуба доказал следующие две теоремы, которые полностью решили проблему Мура по улучшению оценки длины эксперимента в теореме 8 из [11].

Теорема А (А. А. Карацуба). Если $S$ - $(n ; m ; p)$-машина с попарно отличимыми состояниями, то существует разветвленный эксперимент длины не более чем $(n-1)(n-2) / 2+1$, с помощъю которого можно определить (найти) состояние $S$ в конще эксперимента.

Теорема В (А. А. Карацуба). Существует такая $(n ; m ; p)$-машина с попарно отличимыми состояниями, что длина наикратчайшего эксперимента, устанавливающего состояние машины в конце эксперимента, равна $(n-1)(n-2) / 2+1$. 
Теоремы А и В явились основой курсовой работы А. А. Карацубы, которая была отмечена похвальным отзывом (т.е. не очень высоко) на конкурсе студенческих работ механико-математического факультета МГУ им. М. В. Ломоносова в 1958 г. Статья [1] была представлена в журнал «Успехи математических наук» 17 декабря 1958 г., а опубликована лишь в июне 1960 г. Впоследствии этот результат Карацубы стал называться теоремой Myра-Караиубъ.

2.2. Быстрые алгоритмы. Быстрые алгоритмы - это область вычислительной математики, которая изучает алгоритмы вычисления заданной функции с заданной точностью с использованием как можно меньшего числа битовых операций. Будем считать, что числа записаны в двоичной системе счисления, знаки которой 0 и 1 называются битами. Одна битовая операция определяется как запись знаков 0, 1, плюс, минус, скобка, сложение, вычитание и умножение двух битов.

Первые постановки задач о битовой сложности вычисления принадлежат А. Н. Колмогорову. Сложность умножения $M(n)$ определяется как количество битовых операций, достаточное для вычисления произведения двух $n$-значных чисел. Перемножая два $n$-значных числа обычным способом в столбик, мы имеем оценку сверху

$$
M(n)=O\left(n^{2}\right)
$$

В 1956 г. А.Н. Колмогоров высказал гипотезу, что нижняя оценка $M(n)$ при любом методе умножения есть также величина порядка $n^{2}$, т.е. нельзя вычислить произведение двух $n$-значных чисел быстрее, чем за $n^{2}$ операций (так называемая гипотеза $n^{2}$ Колмогорова). На правдоподобность гипотезы $n^{2}$ указывал тот факт, что за все время существования математики к тому моменту люди производили умножение со сложностью порядка $O\left(n^{2}\right)$, и если бы существовал более быстрый метод умножения, то он, вероятно, уже был бы найден.

В 1960 г. А. А. Карацуба нашел новый метод умножения двух $n$-значных чисел, известный теперь как метод или алгоритм умножения Карацубъ, с оценкой сложности

$$
M(n)=O\left(n^{\log _{2} 3}\right)=O\left(n^{1,58496 \ldots}\right)
$$

и тем самым опроверг гипотезу $n^{2}$. Этот результат был доложен Анатолием Карацубой на семинаре А. Н. Колмогорова в МГУ в 1960 г., после чего семинар был Колмогоровым закрыт. Первая статья с описанием этого метода [2] была подготовлена самим Колмогоровым. Там он представил два разных и не связанных друг с другом результата двух своих учеников, и хотя в статье Колмогоров четко отметил, что одна теорема (не связанная с быстрым умножением) принадлежит Ю. Офману, а другая теорема (с первым в истории быстрым умножением) принадлежит А. Карацубе, эта публикация под именами двух авторов надолго сбила с толку читателей, которые полагали, что оба автора внесли вклад в создание быстрого умножения, и даже называли этот метод двумя именами. Метод Карацубы впоследствии получил название "Разделяй и властвуй" (Divide and conquer). Иногда в зависимости от сферы применения он фигурирует также под другими названиями, например, Binary Splitting (“двоичное разбиение") и др.

Впоследствии на основе этой идеи А. А. Карацубы (см. [12], [13]) было построено множество быстрых алгоритмов, самыми известными из которых, по-видимому, являются быстрое преобразование Фуръе (см. [14]), метод умножения Шенхаге-Штрассена (см. [15]) и метод матричного умножения IIтрассена (см. [16]). В последние годы название Divide and Conquer используется для действий с разбиением задачи на части, и это уже не всегда связано с быстрыми вычислительными алгоритмами. 


\section{3. Работы по теории чисел. Тригонометрические суммы и тригонометрические интегралы}

Начало исследованиям Анатолия Алексеевича Карацубы в области теории чисел положили его работы в теории тригонометрических сумм и тригонометрических интегралов.

В 1961 г. А.А. Карацуба поставил и решил задачу, которую можно назвать "аналогом проблемы Варинга для сравнений". Эта задача и метод ее решения привели к созданию нового p-адического метода доказательства теоремы о среднем Виноградова, а затем к созданию общего $p$-адического метода, составившего основу теории кратных тригонометрических сумм (см. [17]-[33], а также [6] и [9]).

3.1. $\boldsymbol{p}$-адический метод А. А. Карацубы и его развитие. Под $p$-адическими методами подразумеваются методы аналитической теории чисел, связанные с использованием различных свойств системы вычетов по степени простого числа $p$. В книге [6, введение, с. 8] (см. также [9]) читаем следующее.

"Применяемый в настоящей книге $p$-адический метод был в основном разработан в 19621966 гг. (см. [39-42] $\left.)^{1}\right)$. В дальнейшем этот метод развивался и совершенствовался (см. [2-28, $\left.43,56-62]^{2}\right)$, и в настоящее время он включает в себя ряд приемов и соображений, тесно связанных между собой в идейном отношении.

Отметим наиболее характерные из них.

1) Использование кругового метода в $p$-адической форме.

2) Применение $p$-адического аналога $u$-чисел И. М. Виноградова, реализация в $p$-адической форме принципа вложения Эйлера-Виноградова при оценке числа решений уравнений и сравнений варинговского типа.

3) Понижение степени многочлена за счет сдвига аргумента (т.е. разбиения значений аргумента на прогрессии) на число, кратное некоторой степени простого.

4) Рекуррентное сведение аддитивных задач на неполную систему вычетов по модулю $p^{n}$ к сравнениям на полную систему вычетов и к задачам того же типа, но с меньшим значением главных и неглавных параметров.

5) Использование условий регулярности решений систем уравнений и сравнений в $p$-адической форме.

6) Применение переменных параметров в рекуррентном процессе пунктов 2)-4) и оптимизация по этим параметрам.

7) Переход от выщербленных систем к полным за счет локального р-адического изменения неизвестных.

8) Одновременное использование нескольких модулей вида $p^{n}$, отвечающих различным простым числам $p$.

9) Использование идеи сглаживания в $p$-адической трактовке.

10) Переход в сравнениях от многочленов к показательным функциям и наоборот.

11) Способы оценок меры множества точек с малым значением функций через значения их параметров (коэффициентов и т.д.) и обратных оценок этих параметров через меру в $p$-адическом и вещественном вариантах; вещественная интерпретация приемов и соображений, изложенных в пунктах 2)-4), 6) и 7)."

С помощью построенного р-адического метода А. А. Карацуба получил ряд новых принципиальных результатов в теории чисел. Доказанные в [17] оценки так называемых $L$-сумм вида

$$
S=\sum_{x=1}^{P} e^{2 \pi i\left(a_{1} x / p^{n}+\cdots+a_{n} x^{n} / p\right)}, \quad\left(a_{s}, p\right)=1, \quad 1 \leqslant s \leqslant n
$$

\footnotetext{
${ }^{1}$ Ссылки соответствуют статьям [20], [24]-[26] в перечне литературы настоящей статьи.

${ }^{2}$ Ссылки на статьи см. в [6], см. также [9].
} 
привели к новым границам нулей $L$-рядов Дирихле по модулю, равному степени простого числа, к выводу асимптотической формулы в [18] для числа решений варинговского сравнения вида

$$
x_{1}^{n}+\cdots+x_{t}^{n} \equiv N\left(\bmod p^{k}\right),
$$

где $n, k, t$ - целые числа, $p$ - простое число, $N$ - произвольное целое число, а $x_{i}, i=1,2, \ldots, t$ пробегают часть полной системы вычетов по модулю $p^{k}$, решению проблемы распределения дробных долей многочлена с целыми коэффициентами по модулю $p^{k}$ (см. [19]). А. А. Карацуба первым реализует (см. [20]) в р-адической форме приниип вложения Эйлера-Виноградова и строит $p$-адический аналог $u$-чисел Виноградова при оценке числа решений сравнения варинговского типа.

Пусть

$$
x_{1}^{n}+\cdots+x_{t}^{n} \equiv N(\bmod Q), \quad 1 \leqslant x_{i} \leqslant P, \quad i=1, \ldots, t,
$$

где $n, N, t, Q, P$ - натуральные числа, причем

$$
P^{r} \leqslant Q<P^{r+1}, \quad 1 \leqslant r \leqslant \frac{1}{12} \sqrt{n}, \quad Q=p^{k}, \quad k \geqslant 4(r+1) n,
$$

где $p$ - простое число. А. А. Карацуба доказал, что в этом случае для всякого натурального числа $n \geqslant 144$ существует $p_{0}=p_{0}(n)$ такое, что для любого $p>p_{0}$ всякое натуральное число $N$ представимо в виде (1) при $t \geqslant 20 r+1$, а при $t<r$ существуют $N$ такие, что сравнение $(1)$ неразрешимо. Этот новый подход, найденный А. А. Карацубой, привел к новому р-адическому доказательству (см. [20]-[22], [25]) теоремы о среднем И. М. Виноградова (см. [33]), играющей центральную роль в методе тригонометрических сумм Виноградова.

В [6] в параграфе III.4 "Новое $p$-адическое доказательство теоремы И. М. Виноградова о среднем", читаем: "И. М. Виноградов от своего метода оценки $G(n)^{3}$ пришел к новому методу оценок сумм Г. Вейля, применив лемму о попаданиях к доказательству теоремы о среднем. Подобным образом А. А. Карацуба от своего аналога проблемы Варинга для сравнений пришел к новому p-адическому методу доказательства теоремы И. М. Виноградова о среднем. Здесь аналогом леммы о попаданиях служит лемма Ю. В. Линника о числе решений полной системы сравнений". Подобная лемма применялась Ю. В. Линником (см. [34]) в его p-адическом доказательстве теоремы И. М. Виноградова о среднем (в ослабленной форме).

Еще одним элементом p-адического метода А.А. Карацубы является переход от неполных систем уравнений к полным за счет локального $p$-адического изменения неизвестных (см. [23], [24]). Пусть $r$ - произвольное натуральное число, $1 \leqslant r \leqslant n$, и целое число $t$ определяется неравенствами $m_{t} \leqslant r<m_{t+1}$. Рассмотрим систему уравнений

$$
\begin{aligned}
& \left\{\begin{array}{c}
x_{1}^{m_{1}}+\cdots+x_{k}^{m_{1}}=y_{1}^{m_{1}}+\cdots+y_{k}^{m_{1}}, \\
\ldots \ldots \ldots \ldots \cdots \cdots \cdots \cdots+x_{k}^{m_{s}}=y_{1}^{m_{s}}+\cdots+y_{k}^{m_{s}}, \\
x_{1}^{m_{s}}+\cdots+x_{k}^{n}=y_{1}^{n}+\cdots+y_{k}^{n}, \\
x_{1}^{n}+\cdots+
\end{array}\right. \\
& 1 \leqslant x_{1}, \ldots, x_{k}, y_{1}, \ldots, y_{k} \leqslant P, \quad 1 \leqslant m_{1}<m_{2}<\cdots<m_{s}<m_{s+1}=n \text {. }
\end{aligned}
$$

А. А. Карацуба доказал, что для числа решений $I_{k}$ этой системы уравнений при $k \geqslant 6 r n \log n$ справедлива оценка

$$
I_{k} \ll P^{2 k-\delta}, \quad \delta=m_{1}+\cdots+m_{t}+(s-t+1) r .
$$

Для неполных систем уравнений, в которых переменные пробегают числа с малыми простыми делителями, А. А. Карацуба применил мультипликативный сдвиг переменных. Это привело к качественно новой оценке тригонометрических сумм и новой теореме о среднем для таких систем уравнений (см. [25]).

\footnotetext{
${ }^{3}$ Функция Харди-Литтлвуда в проблеме Варинга.
} 
3.2. Кратные тригонометрические суммы. В 1961-1966 гг. А. А. Карацуба заложил (см. [17]-[28]) основы теории кратных тригонометрических сумм Германа Вейля, т.е. сумм вида

$$
S=S(A)=\sum_{x_{1}=1}^{P_{1}} \cdots \sum_{x_{r}=1}^{P_{r}} e^{2 \pi i F\left(x_{1}, \ldots, x_{r}\right)},
$$

где

$$
F\left(x_{1}, \ldots, x_{r}\right)=\sum_{t_{1}=1}^{n_{1}} \cdots \sum_{t_{r}=1}^{n_{r}} \alpha\left(t_{1}, \ldots, t_{r}\right) x_{1}^{t_{1}} \cdots x_{r}^{t_{r}},
$$

$A$ - набор вещественных коэффициентов $\alpha\left(t_{1}, \ldots, t_{r}\right)$. В частности, он сформулировал теоремы об оценке среднего значения кратной тригонометрической суммы $S(A)$. Принципиальная возможность получения требуемой оценки открылась после разработки А. А. Карацубой p-адического варианта метода И. М. Виноградова. Тогда же были получены первые грубые оценки сумм $S(A)$. В 1971 г. ученик Карацубы Г. И. Архипов (опубликовано в 1975 г. в [35]) получил оценки двукратных тригонометрических сумм, подобные оценкам Виноградова. Наконец, к началу 1980-х годов А. А. Карацуба и его ученики Г. И. Архипов и В. Н. Чубариков (см. [29]-[33], а также [6], [9]) завершили в основном построение всей теории кратных сумм Г. Вейля (без ограничения на их кратность).

Центральным моментом этой теории, как и теории тригонометрических сумм И. М. Виноградова, является следующая теорема.

ТЕОРЕМА О СРЕДНЕМ. Пустъ $n_{1}, \ldots, n_{r}, P_{1}, \ldots, P_{r}-$ натуральнъе числа,

$$
P_{1}=\min \left(P_{1}, \ldots, P_{r}\right), \quad m=\left(n_{1}+1\right) \cdots\left(n_{r}+1\right) .
$$

Пусть, далее, $\Omega$ - т-мерный куб в евклидовом пространстве вида

$$
0 \leqslant \alpha\left(t_{1}, \ldots, t_{r}\right)<1, \quad 0 \leqslant t_{1} \leqslant n_{1}, \quad \ldots, \quad 0 \leqslant t_{r} \leqslant n_{r}
$$

$u$

$$
J=J\left(P_{1}, \ldots, P_{r} ; n_{1}, \ldots, n_{r} ; K, r\right)=\int \ldots \int|S(A)|^{2 K} d A .
$$

Тогда при любом $\tau \geqslant 0$ и $K \geqslant K_{\tau}=m \tau$ для величины $J$ имеет место оченка

$$
J \leqslant K_{\tau}^{2 m \tau} \varkappa^{4 \varkappa^{2} \Delta(\tau)} 2^{8 m \varkappa \tau}\left(P_{1} \cdots P_{r}\right)^{2 K} P^{-\varkappa \Delta(\tau)},
$$

¿de

$$
\varkappa=n_{1} \nu_{1}+\cdots+n_{r} \nu_{r}, \quad \gamma \varkappa=1, \quad \Delta(\tau)=\frac{m}{2}\left(1-(1-\gamma)^{\tau}\right), \quad P=\left(P_{1}^{n_{1}} \cdots P_{r}^{n_{r}}\right)^{\gamma},
$$

u натуральные числа $\nu_{1}, \ldots, \nu_{r}$ таковы, что

$$
-1<\frac{\ln P_{s}}{\ln P_{1}}-\nu_{s} \leqslant 0, \quad s=1, \ldots, r .
$$

Теорема о среднем и лемма о кратности пересечения многомерных параллелепипедов лежат в основе оценки кратной тригонометрической суммы (см. [25], [31], а также [6], [9]). 
3.3. Проблема Хуа Ло-кена о показателе сходимости особого интеграла проблемы Терри. -Адический метод А.А. Карацубы включает в себя способы оценок меры множества точек с малыми значениями функций через значения их параметров (коэффициенты и т.п.) и, обратно, оценок этих параметров через меру множества в вещественной и p-адической метриках. Особенно ярко эта сторона метода А. А. Карацубы проявилась при оценках тригонометрических интегралов, что привело к решению проблемы Хуа Ло-кена. В 1979 г. А. А. Карацуба вместе со своими учениками Г. И. Архиповым и В. Н. Чубариковым полностью решили (см. [36]) проблему Хуа Ло-кена, поставленную в 1937 г., которая заключалась в определении показателя сходимости интеграла

$$
\vartheta_{0}=\int_{-\infty}^{+\infty} \cdots \int_{-\infty}^{+\infty}\left|\int_{0}^{1} e^{2 \pi i\left(\alpha_{n} x^{n}+\cdots+\alpha_{1} x\right)} d x\right|^{2 k} d \alpha_{n} \ldots d \alpha_{1}
$$

которым называется такое число $\gamma$, что $\vartheta_{0}$ сходится при $2 k>\gamma+\varepsilon$ и расходится при $2 k<\gamma-\varepsilon$, где $\varepsilon>0$ произвольное сколь угодно малое число.

Было установлено, что интеграл $\vartheta_{0}$ сходится при $2 k>\left(n^{2}+n\right) / 2+1$ и расходится при $2 k \leqslant\left(n^{2}+n\right) / 2+1$. Тогда же была решена и аналогичная проблема для интеграла

$$
\vartheta_{1}=\int_{-\infty}^{+\infty} \cdots \int_{-\infty}^{+\infty}\left|\int_{0}^{1} e^{2 \pi i\left(\alpha_{n} x^{n}+\alpha_{m} x^{m}+\cdots+\alpha_{r} x^{r}\right)} d x\right|^{2 k} d \alpha_{n} d \alpha_{m} \ldots d \alpha_{r}
$$

где $n, m, \ldots, r$ - целые числа, удовлетворяющие условиям

$$
1 \leqslant r<\cdots<m<n, \quad r+\cdots+m+n<\frac{1}{2}\left(n^{2}+n\right) .
$$

А.А. Карацубой и его учениками было установлено, что интеграл $\vartheta_{1}$ сходится, если $2 k>$ $n+m+\cdots+r$ и расходится, если $2 k \leqslant n+m+\cdots+r$.

Интегралы $\vartheta_{0}$ и $\vartheta_{1}$ возникают при решении проблемы Терри (см., например, [37]). А. А. Карацубой и его учениками был получен ряд новых результатов, связанных с многомерным аналогом проблемы Терри. В частности, ими было установлено, что если $F$ - полином от $r$ переменных $(r \geqslant 2)$ вида

$$
F\left(x_{1}, \ldots, x_{r}\right)=\sum_{\nu_{1}=0}^{n_{1}} \cdots \sum_{\nu_{r}=0}^{n_{r}} \alpha\left(\nu_{1}, \ldots, \nu_{r}\right) x_{1}^{\nu_{1}} \cdots x_{r}^{\nu_{r}},
$$

с нулевым свободным коэффициентом, $m=\left(n_{1}+1\right) \cdots\left(n_{r}+1\right)-1$, а $\bar{\alpha}-m$-мерный вектор, координатами которого являются коэффициенты многочлена $F\left(x_{1}, \ldots, x_{r}\right)$, то интеграл

$$
\vartheta_{2}=\int_{-\infty}^{+\infty} \cdots \int_{-\infty}^{+\infty}\left|\int_{0}^{1} \cdots \int_{0}^{1} e^{2 \pi i F\left(x_{1}, \ldots, x_{r}\right)} d x_{1} \ldots d x_{r}\right|^{2 k} d \bar{\alpha}
$$

сходится при $2 k>n m$, где $n=\max \left(n_{1}, \ldots, n_{r}\right)$. Этот результат, не являясь окончательным, породил новое направление в теории тригонометрических интегралов, связанное с уточнением границ для показателя сходимости $\vartheta_{2}$ (см., например, [38]).

3.4. Оценка функции Харди в проблеме Варинга.Применяя сконструированную им p-адическую форму кругового метода Харди-Литтлвуда-Рамануджана-Виноградова к оценкам тригонометрических сумм, в которых суммирование ведется по числам с малыми простыми делителями, А. А. Карацуба получил в [39] новую оценку известной функции Харди $G(n)$ в проблеме Варинга (при $n \geqslant 400)$

$$
G(n)<2 n \log n+2 n \log \log n+12 n .
$$


3.5. Проблема Артина о локальном представлении нуля формой. В исследованиях по проблеме Артина о $p$-адическом представлении нуля формой произвольной степени результаты А. А. Карацубы показали, что вместо ранее предполагавшегося степенного роста числа переменных для нетривиального представления нуля формой это число переменных должно расти почти экспоненциально в зависимости от степени. А. А. Карацуба вместе с Г. И. Архиповым доказали в [40], что для любого натурального числа $r$ существует такое $n_{0}=n_{0}(r)$, что для любого $n \geqslant n_{0}$ существует форма $F\left(x_{1}, \ldots, x_{k}\right)$ степени, меньшей $n$, с целыми коэффициентами, число переменных которой $k, k \geqslant 2^{u}$,

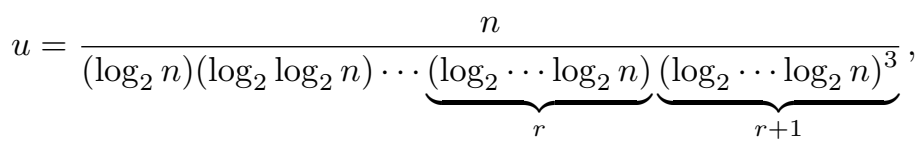

и имеющая только тривиальное представление нуля в 2-адических числах, а также получили аналогичный результат для произвольного нечетного простого модуля $p$.

3.6. Оценки коротких сумм Клоостермана. А. А. Карацуба создал (см. [41]-[45], а также [9]) новый метод оценок коротких сумм Клоостермана, т.е. тригонометрических сумм вида

$$
\sum_{n \in A} \exp \left(2 \pi i \frac{a n^{*}+b n}{m}\right)
$$

где $n$ пробегает некоторое множество $A$ чисел, взаимно простых с $m$, число $\|A\|$ элементов в котором существенно меньше $m$, а символ $n^{*}$ обозначает вычет, обратный к $n$ по модулю $m$ : $n n^{*} \equiv 1(\bmod m)$.

Суммы Клоостермана изучались в работах Г. Клоостермана, Г. Вейля, И. М. Виноградова, Г. Хассе, А. Вейля, Х. Салье, К. Хооли, Л. Карлица, С. Учиямы, В. Шмидта, Р. Бейкера и многих других (см., например, [37]), но до начала 1990-х годов оценки такого типа были известны в основном для сумм, число слагаемых в которых превосходит $\sqrt{m}$. Исключение составляли специальные модули вида $m=p^{\alpha}$, где $p$ - фиксированное простое число, а показатель $\alpha$ неограниченно возрастает (этот случай был исследован А. Г. Постниковым методом И. М. Виноградова).

Метод А. А. Карацубы позволяет оценивать суммы Клоостермана, число слагаемых которых не превосходит $m^{\varepsilon}$, а в некоторых случаях - даже $\exp \left\{(\ln m)^{2 / 3+\varepsilon}\right\}$, где $\varepsilon>0-$ сколь угодно малое фиксированное число.

Различные аспекты метода А. А. Карацубы нашли применение в решении следующих задач аналитической теории чисел:

• нахождение нижней границы для числа решений неравенств вида

$$
\alpha<\left\{\frac{a n^{*}+b n}{m}\right\} \leqslant \beta
$$

в целых числах $n, 1 \leqslant n \leqslant x$, взаимно простых с $m, x<\sqrt{m}$ (А. А. Карацуба [41], [44]); - нахождение асимптотик сумм дробных долей вида

$$
\sum_{n \leqslant x},\left\{\frac{a n^{*}+b n}{m}\right\}, \quad \sum_{p \leqslant x},\left\{\frac{a p^{*}+b p}{m}\right\},
$$

где $n$ пробегает подряд идущие целые числа с условием $(n, m)=1$, а $p$ пробегает простые числа, не делящие модуль $m$ (А. А. Карацуба [44]);

- точность приближения произвольного вещественного числа из отрезка $[0,1]$ дробными долями вида

$$
\left\{\frac{a n^{*}+b n}{m}\right\}
$$

где $1 \leqslant n \leqslant x,(n, m)=1, x<\sqrt{m}$ (А. А. Карацуба [40], [42]); 
- уточнение постоянной $c$ в неравенстве Бруна-Титчмарша

$$
\pi(x ; q, l)<\frac{c x}{\varphi(q) \ln (2 x / q)},
$$

где $\pi(x ; q, l)$ - число простых чисел $p$, не превосходящих $x$ и принадлежащих арифметической прогрессии $p \equiv l(\bmod q)$ (Дж. Фридлендер, Х. Иванец [46]);

- доказательство бесконечности простых чисел вида $a^{2}+b^{4}$ (Дж. Фридлндер, Х. Иванец [47]);

- нижняя оценка наибольшего простого делителя произведения чисел вида $n^{3}+2, N<$ $n \leqslant 2 N$ (Д. Р. Хиз-Браун [48]);

- комбинаторные свойства множества чисел $n^{*}(\bmod m), 1 \leqslant n \leqslant m^{\varepsilon}$ (А. А. Глибичук [49]).

\section{4. Работы по теории чисел. Дзета-функция Римана}

4.1. Гипотеза А. Сельберга. В 1983 г. А. А. Карацуба установил (см. [50]-[52]), что при произвольном положительном $\varepsilon$, не превосходящем 0.001 ,

$$
T \geqslant T_{0}(\varepsilon)>0, \quad H=T^{a+\varepsilon}, \quad a=\frac{27}{82}=\frac{1}{3}-\frac{1}{246},
$$

промежуток $(T, T+H)$ содержит не менее $c H \ln T$ вещественных нулей нечетного порядка дзета-функции Римана $\zeta(1 / 2+i t)$, где $c=c(\varepsilon)>0$ - некоторая постоянная.

Это утверждение в 1942 г. было высказано в качестве гипотезы А. Сельбергом (см. [53]), который сам доказал его справедливость для случая $H \geqslant T^{1 / 2+\varepsilon}$. Оценки А. Сельберга и А. А. Карацубы являются неулучшаемыми по порядку роста при $T \rightarrow+\infty$.

4.2. Распределение нулей дзета-функции Римана на коротких отрезках критической прямой. А. А. Карацубе принадлежит также ряд результатов о распределении нулей $\zeta(s)$ на коротких промежутках критической прямой. Он доказал, что аналог гипотезы Сельберга справедлив для почти всех промежутков $(T, T+H), H=T^{\varepsilon}$, где $\varepsilon-$ сколь угодно малое фиксированное положительное число.

В 1992 г. А.А. Карацуба разработал (см. [54]) новый подход к исследованию нулей дзета-функции Римана на сверхкоротких промежутках критической прямой, т.е. на промежутках $(T, T+H)$, длина $H$ которых растет медленнее любой, даже сколь угодно малой степени $T$. В частности, он доказал, что для любых сколь угодно малых фиксированных чисел $\varepsilon$, $\varepsilon_{1}$ с условием $0<\varepsilon, \varepsilon_{1} \leqslant 1$ при

$$
\exp \left(\log ^{\varepsilon} Y\right) \leqslant H \leqslant Y^{1 / 3}, \quad Y \geqslant Y_{1}\left(\varepsilon, \varepsilon_{1}\right)>0
$$

для почти всех $T \in(Y, 2 Y)$ число нулей нечетного порядка функции $\zeta(1 / 2+i t)$ на промежутке $(T, T+H)$ не меньше чем

$$
H(\ln H)^{1-\varepsilon_{1}} .
$$

Эта оценка весьма близка к той, что следует из гипотезы Римана.

4.3. Функция Харди в теории дзета-функции Римана. При изучении нулей $\zeta(s)$, лежащих на критической прямой, вместо $\zeta(s)$ часто удобно иметь дело с функцией Харди

$$
Z(t)=\pi^{-i t / 2} \frac{\Gamma(1 / 4+i t / 2)}{|\Gamma(1 / 4+i t / 2)|} \zeta\left(\frac{1}{2}+i t\right)
$$

Эта функция вещественна при вещественных $t$, а ее вещественные нули совпадают с ординатами нулей $\zeta(s)$, лежащих на критической прямой. В 1918 г. Г. Х. Харди и Дж. И. Литтлвуд 
(см. [55]) доказали, что всякий промежуток $(T, T+H)$ при $H \geqslant T^{1 / 4+\varepsilon}$ и $T \geqslant T_{0}(\varepsilon)>0$ содержит по крайней мере один нуль $Z(t)$ нечетного порядка, откуда

$$
N_{0}(T+H)-N_{0}(T)>0
$$

где $N_{0}(T)$ - число нулей $\zeta(1 / 2+i t)$ при $0<t \leqslant T$. В 1981 г. Я. Мозер доказал в [56] оценку $(2)$ для $H \geqslant T^{1 / 6+1 / \psi}$, где

$$
0<\psi(T)<\frac{1}{6} \frac{\ln T}{\ln \ln T}, \quad T \rightarrow+\infty .
$$

Особенность рассматриваемой задачи заключается в том, что верхняя и нижняя границы промежутка суммирования в сумме $S$ имеют один и тот же порядок $P$, в то время как число слагаемых в $S$ имеет порядок существенно меньший: $o(P)$. Занимаясь нулями функции Харди, А. А. Карацуба сумел удачно использовать эту особенность и в 1981 г. получил (см. [57]) оценку суммы $S$, более точную, чем оценка Мозера. Следствием этого результата явилось существование нуля нечетного порядка функции $Z(t)$ на промежутке $(T, T+H)$, где

$$
H=T^{5 / 32} \ln ^{2} T=T^{1 / 6-1 / 96} \ln ^{2} T, \quad T \geqslant T_{0}>0 .
$$

Обобщив впоследствии этот прием, А. А. Карацуба успешно применил его к новой, никем до того не исследованной задаче о вещественных нулях производных функции Харди (см. [58]). При изучении этого вопроса им было обнаружено неожиданное явление, получившее впоследствии название эфбекта сближения нулей у функций $Z^{(k)}(t)$. Оказалось, что по мере роста порядка $k$ производной длина $H$ промежутка $(T, T+H)$, на котором можно "уловить" нуль нечетного порядка $Z^{(k)}(t)$, не увеличивается, как можно было бы ожидать, а, напротив, уменьшается и составляет величину порядка

$$
T^{1 /(6 k+6)}(\ln T)^{2 /(k+1)},
$$

где $k$ - натуральное число, $T \geqslant T_{0}>0$. Впоследствии ученица А. А. Карацубы А. А. Лаврик распространила (см. [59]) этот результат на случай растущих вместе с $T$ значений $k$.

4.4. Плотностные теоремы для дзета-функции Римана и аргумент дзета-функции Римана. Еще один подход к изучению нулей дзета-функции Римана в критической полосе состоит в нахождении верхних оценок для величины $N(\sigma, T)$ - числа нулей $\zeta(s)$ в прямоугольнике $\sigma<\operatorname{Re} s \leqslant 1,0<\operatorname{Im} s \leqslant T$, где $1 / 2 \leqslant \sigma \leqslant 1$. Такие оценки называются nлотностными теоремами. Хотя гипотеза Римана влечет при любых $\sigma>1 / 2$ и $T>0$ равенство $N(\sigma, T)=0$, в ряде случаев плотностные теоремы позволяют доказывать утверждения, близкие к следствиям из гипотезы Римана. Плотностные теоремы используются для изучения свойств функции

$$
S(t)=\frac{1}{\pi} \arg \zeta\left(\frac{1}{2}+i t\right),
$$

которая называется аргументом дзета-функции Римана на критической прямой (здесь $\arg \zeta(1 / 2+i t)$ - приращение произвольной непрерывной ветви $\arg \zeta(s)$ вдоль ломаной линии, соединяющей точки $2,2+i t$ и $1 / 2+i t)$. Несмотря на то, что было давно известно, что $S(t)$ бесконечно много раз меняет свой знак, А. Сельберг (см. [60]) был первым, кто смог получить нижнюю оценку для числа перемен знака $S(t)$ на заданном промежутке $(T, T+H)$ при $H \geqslant T^{1 / 2+\varepsilon}$. Однако, для случая $H<\sqrt{T}$ получение подобных оценок оставалось открытой проблемой.

А. А. Карацубе удалось доказать (см. [61], [62]) ряд теорем о функции $S(t)$ для случая "коротких" промежутков $(T, T+H]$, где $H=T^{27 / 82+\varepsilon}$. В их числе - теоремы о средних значениях функции $S(t)$ и ее первообразной $S_{1}(t)=\int_{0}^{t} S(u) d u$ на отрезках вещественной 
прямой, а также теорема о том, что всякий промежуток $(T, T+H]$ при $H \geqslant T^{27 / 82+\varepsilon}$ содержит не менее

$$
H(\ln T)^{1 / 3} e^{-c \sqrt{\ln \ln T}}
$$

точек перемены знака функции $S(t)$.

4.5. Омега-теоремы для дзетовых сумм. По предложению А.А. Карацубы тригонометрические суммы

$$
S=\sum_{n \leqslant N} n^{i t}, \quad S_{1}=\sum_{a<n \leqslant b} n^{i t},
$$

где $t$ - растущий параметр, $t \geqslant t_{1}>0, N=N(t) \leqslant t, a<b \leqslant 2 a \leqslant t$, называются дзетовыми. Верхние оценки абсолютных значений дзетовых сумм тесно связаны с задачей о порядке роста величины $|\zeta(\sigma+i t)|$ при $t \rightarrow+\infty$, где $\sigma$ - фиксированное число, $1 / 2 \leqslant \sigma<1$. Например, гипотеза Линделефа (см., например, [7]), согласно которой справедливо соотношение

$$
\zeta\left(\frac{1}{2}+i t\right)=O\left(|t|^{\varepsilon}\right), \quad|t| \geqslant 2,
$$

и оценка дзетовой суммы вида

$$
|S| \ll \sqrt{N}|t|^{\varepsilon}, \quad 1 \leqslant N \leqslant \sqrt{|t|},
$$

являются эквивалентными утверждениями.

А. А. Карацуба доказал теорему (см. [63]) о том, что существует бесконечная последовательность чисел $t_{\nu}, \nu=1,2,3, \ldots$, таких, что $t_{1} \geqslant 1, t_{\nu+1} t_{\nu}^{-1} \geqslant 2$, и обладающих следующим свойством: для всякого $t=t_{\nu}$ на интервале $(1,[\sqrt{t /(2 \pi)}])$ найдется по крайней мере $K$ натуральных чисел $N, K \geqslant f^{2}(t)$, где

$$
f(t)=\exp \left(\frac{3}{5} \sqrt{\frac{\ln t}{\ln \ln t}}\right)
$$

таких, что

$$
\left|\sum_{n=1}^{N} n^{i t}\right| \geqslant \sqrt{N} f(t) .
$$

Из этой теоремы следует, что, по-видимому, нельзя получить оценку дзетовой суммы, лучшую, чем, например,

$$
|S(N)| \leqslant \sqrt{N} \exp \left(c \sqrt{\frac{\ln t}{\ln \ln t}}\right),
$$

где $c>0$ - абсолютная постоянная. Верхние оценки $|S(N)|$, которые получаются даже из гипотезы Римана сильно отличаются от (3). При справедливости гипотезы Римана имеет место оценка

$$
|S(N)| \leqslant \sqrt{N} \exp \left(c \frac{\ln t}{\ln \ln t}\right),
$$

где $c>0$ - абсолютная постоянная, $N \leqslant t$.

4.6. Нули линейных комбинаций $L$-рядов Дирихле. А. А. Карацубой создан новый метод (см. [64]) исследования нулей функций, представимых в виде линейных комбинаций $L$-рядов Дирихле. Простейшим примером функции такого рода служит функция Дэвенпорта-Хейльбронна (см. [65]), определяемая равенством

$$
f(s)=\frac{1}{2}(1-i \varkappa) L\left(s, \chi_{1}\right)+\frac{1}{2}(1+i \varkappa) L\left(s, \bar{\chi}_{1}\right),
$$


где $\chi_{1}(n)$ - комплексный характер по модулю 5, т.е.

$$
\begin{gathered}
\chi_{1}(1)=1, \quad \chi_{1}(2)=i, \quad \chi_{1}(3)=-i, \quad \chi_{1}(4)=-1, \quad \chi_{1}(5)=0, \\
\chi_{1}(n+5)=\chi_{1}(n) \quad \text { для любого } n, \\
L(s, \chi)=\sum_{n=1}^{\infty} \frac{\chi(n)}{n^{s}}, \quad \varkappa=\frac{\sqrt{10-2 \sqrt{5}}-2}{\sqrt{5}-1} .
\end{gathered}
$$

Для $f(s)$ гипотеза Римана неверна, однако критическая прямая $\operatorname{Re} s=1 / 2$ содержит, тем не менее, аномально много нулей, как установил ученик А. А. Карацубы С. М. Воронин (см. [66]).

В 1989 г. А. А. Карацуба доказал (см. [67], а также [64], [68]), что промежуток (T, $T+H]$, $H=T^{27 / 82+\varepsilon_{1}}, T \geqslant T_{0}\left(\varepsilon, \varepsilon_{1}\right)>0$, содержит не менее

$$
H(\ln T)^{1 / 2-\varepsilon}
$$

нулей нечетного порядка функции $f(1 / 2+i t)$. Здесь $\varepsilon, \varepsilon_{1}-$ произвольно малые фиксированные положительные числа, не превосходящие 0.01. Отсюда следует (см. [68]), что если $N_{1}(T)-$ количество нулей $\rho$ нечетного порядка функции Дэвенпорта-Хейльбронна $f(s)$ с условием $\operatorname{Re} \rho=1 / 2,0<\operatorname{Im} \rho \leqslant T$, то справедлива оценка снизу величины $N_{1}(T)$ :

$$
N_{1}(T) \geqslant T(\ln T)^{1 / 2} \exp \left(-a_{1} \sqrt{\ln \ln T}\right),
$$

где $a_{1} \geqslant 1$ - абсолютная постоянная, $T \geqslant T_{1}>0$.

Подобные результаты были получены А.А. Карацубой (см. [69]) и для линейных комбинаций $L$-рядов Дирихле, содержащих произвольное (конечное) число слагаемых; показатель степени $1 / 2$ заменяется при этом меньшим числом $\beta$, зависящим лишь от вида линейной комбинации.

4.7. Граница нулей дзета-функции и многомерная проблема делителей Дирихле. А. А. Карацубе принадлежит принципиально новый результат (см. [70]) в многомерной проблеме делителей Дирихле, которая связана с нахождением при $x \rightarrow+\infty$ числа $T_{k}(x)$ решений неравенства $x_{1} \cdots x_{k} \leqslant x$ в натуральных числах $x_{1}, \ldots, x_{k}$. Известно (см., например, $[71])$, что

$$
T_{k}(x)=x P_{k-1}(\ln x)+R_{k}(x),
$$

где $P_{k-1}(u)$ - многочлен степени не больше, чем $k-1$, коэффициенты которого зависят от $k$ и могут быть найдены явно, а $R_{k}(x)$ - остаточный член, причем

$$
\left|R_{k}(x)\right| \leqslant x^{1-\alpha(k)}(c \ln x)^{k},
$$

где $\alpha(k)=1 /(a k+b), a, b, c$ - абсолютные положительные постоянные.

В работе [70] А. А. Карацуба получил более точную оценку $R_{k}(x)$, в которой величина $\alpha(k)$ имела порядок $k^{-2 / 3}$ и убывала гораздо медленнее, чем $\alpha(k)$ в предыдущих оценках. Оценка А. А. Карацубы является равномерной по $x$ и $k$; в частности, величина $k$ может расти по мере роста $x$ (как некоторая степень логарифма $x$ ). Похожий, но более слабый результат был получен в 1960 г. немецким математиком Х.Э. Рихертом (см. [72]), работа которого долгое время (до 1985 г.) оставалась неизвестной российским математикам.

В 2000 г. А. А. Карацуба установил (см. [73], [74]) обратную связь оценок величин $R_{k}(x)$ с поведением $\zeta(s)$ вблизи прямой $\operatorname{Re} s=1$. В частности, он доказал следующие две теоремы.

Теорема С. Пусть $\alpha(y)$ - невозрастающая функиия, $y \geqslant 2,1 / y \leqslant \alpha(y) \leqslant 1 / 2, u$ пусть при некотором $c \geqslant 2$ и всех $k \geqslant 2, x \geqslant 2$ выполняется неравенство

$$
\left|R_{k}(x)\right| \leqslant x^{1-\alpha(k)}(c \ln x)^{k} .
$$


Тогда существует абсолютная постоянная $c_{1}>0$ такая, что в области s-плоскости вида $|t| \geqslant e^{2}$

$$
\operatorname{Re} s \geqslant 1-c_{1} \alpha(\ln |t|)(\ln \ln |t|)^{-1}
$$

бункция $\zeta(s)$ не имеет нулей.

ТеОрема D. Пусть выполняются условия теоремы C. Тогда $\zeta(s) \neq 0$ в области s-nлоскости вида $|t| \geqslant e^{10}$,

$$
\operatorname{Re} s \geqslant 1-c_{1} \alpha\left(\frac{\ln |t|}{\ln \ln |t|}\right)(\ln \ln |t|)^{-1},
$$

где $c_{1}>0$ - абсолютная постоянная.

4.8. Нижние оценки максимума модуля дзета-функции в малых областях критической полосы и на малых промежутках критической прямой. А. А. Карацубой введены и исследованы (см. [75], [76]) функции $F(T ; H)$ и $G\left(s_{0} ; \Delta\right)$, определяемые равенствами

$$
F(T ; H)=\max _{|T-t| \leqslant H}\left|\zeta\left(\frac{1}{2}+i t\right)\right|, \quad G(T ; H)=\max _{|s-(1 / 2+i T)| \leqslant H}|\zeta(s)| .
$$

Здесь $T \geqslant T_{1}>0, H>0$.

Нижние оценки величин $F$ и $G$ показывают, насколько большие (по абсолютной величине) значения может принимать $\zeta(s)$ на коротких отрезках критической прямой или в малых окрестностях точек, лежащих в критической полосе $0 \leqslant \operatorname{Re} s \leqslant 1$. При больших $H$, а именно при $c \ln \ln T \leqslant H \leqslant 0.1 T$, нижняя оценка $F(T, H)$ была получена в [77]; при $\Delta>1 / 2$ задача о нижней оценке $G(T ; \Delta)$ становится тривиальной.

А. А. Карацуба доказал, что

$$
\begin{array}{ll}
F(T ; \Delta) \geqslant e^{A \ln \Delta \ln T}, & \text { если } \quad 0<\Delta \leqslant \frac{1}{\ln T}, \\
G(T ; \Delta) \geqslant e^{6 \ln \Delta \ln T}, & \text { если } \quad 0<\Delta \leqslant \frac{1}{3},
\end{array}
$$

где $A, c$ - абсолютные положительные постоянные. А. А. Карацуба доказал также, что существуют абсолютные постоянные $c>0$ и $T_{1}>0$ такие, что при $T \geqslant T_{1}, 2 \leqslant H \leqslant \ln \ln T-c$ справедлива оценка

$$
F(T ; H) \geqslant \frac{1}{8} T^{-1 /(2(\operatorname{ch} H-1))} .
$$

Здесь $\operatorname{ch} H$ - гиперболический косинус $H$.

\section{5. Работы по теории чисел. Характеры Дирихле}

5.1. Оценки коротких сумм характеров в конечных полях. В конце 1960-х годов А.А. Карацуба, занимаясь оценками коротких сумм характеров Дирихле, создал (см. [78], [79]) новый метод, позволивший получать нетривиальные оценки коротких сумм характеров в конечных полях. Пусть $n \geqslant 1$ - фиксированное целое число,

$$
F(x)=x^{n}+a_{n-1} x^{n-1}+\cdots+a_{1} x+a_{0}
$$

- неприводимый над полем $\mathbb{Q}$ рациональных чисел многочлен, $\theta$ - корень уравнения $F(\theta)=0$, $\mathbb{Q}(\theta)$ - расширение поля $\mathbb{Q}, \omega_{1}, \ldots, \omega_{n}-$ базис $\mathbb{Q}(\theta)$,

$$
\omega_{1}=1, \quad \omega_{2}=\theta, \quad \omega_{3}=\theta^{2}, \quad \ldots, \quad \omega_{n}=\theta^{n-1} .
$$


Пусть, далее, $p$ - достаточно большое простое число такое, что $F(x)$ неприводим по модулю $p$, $\mathrm{GF}\left(p^{n}\right)$ - поле Галуа с базисом $\omega_{1}, \omega_{2}, \ldots, \omega_{n}$, а $\chi$ - неглавный характер Дирихле поля $\mathrm{GF}\left(p^{n}\right)$. Пусть, наконец, $\nu_{1}, \ldots, \nu_{n}$ - некоторые неотрицательные целые числа, $D(X)$ - множество элементов $\bar{x}$ поля Галуа $\mathrm{GF}\left(p^{n}\right), \bar{x}=x_{1} \omega_{1}+\cdots+x_{n} \omega_{n}$, таких, что при любом $i, 1 \leqslant i \leqslant n$, справедливы неравенства

$$
\nu_{i}<x_{i}<\nu_{i}+X
$$

А.А. Карацуба доказал, что при любом фиксированном $k \geqslant n+1$ и произвольном $X$ с условием

$$
p^{1 / 4+1 /(4 k)} \leqslant X \leqslant p^{1 / 2+1 /(4 k)}
$$

справедлива оценка

$$
\left|\sum_{\bar{x} \in D(X)} \chi(\bar{x})\right| \leqslant C\left(X^{1-1 / k} p^{1 /(4 k)+1 /\left(4 k^{2}\right)}\right)^{n}(\ln p)^{\gamma},
$$

где $\gamma=\left(2^{n+1}-1\right) / k, C=C(n, \chi)$.

5.2. Оценки линейных сумм характеров по сдвинутым простым числам.А. А. Карацуба разработал ряд новых приемов, применение которых наряду с методом И. М. Виноградова к оценке сумм характеров с простыми числами позволило ему в 1970 г. получить (см. [80]) оценку суммы значений неглавного характера по простому модулю $q$ на последовательности сдвинутых простых чисел, а именно оценку вида

$$
\left|\sum_{p \leqslant N} \chi(p+k)\right| \ll N q^{-\omega^{2} / 1024},
$$

где $k$ - целое число с условием $k \not \equiv 0(\bmod q), \omega-$ произвольное число из интервала $0<\omega \leqslant$ $1 / 4, q^{1 / 2+\omega} \leqslant N<q$, а постоянная в знаке « зависит лишь от $\omega ;$ при этом число квадратичных вычетов (как и число квадратичных невычетов) по модулю $q$ вида $p+k, p \leqslant N$, равно

$$
\frac{1}{2} \pi(N)+O\left(N q^{-\omega^{2} / 1024}\right)
$$

Соотношение (4) представляет собой значительное усиление оценки И. М. Виноградова (см. [81]), нетривиальной при $N \geqslant q^{3 / 4+\omega}$. Заметим, что оценка эта имеет принципиальное значение, “так как по глубине превосходит то, что дает непосредственное применение расширенной гипотезы Римана, и, по-видимому, в этом направлении является истиной, более глубокой, чем указанная гипотеза (если гипотеза верна)" (Ю.В.Линник, 1971 г., Международная конференция по теории чисел, посвященная 80-летию со дня рождения И. М. Виноградова, см. [10]).

Этот результат был перенесен А.А. Карацубой (см. [82]) и на случай, когда $p$ пробегает простые числа арифметической прогрессии, разность которой растет вместе с модулем $q$.

5.3. Тернарные мультипликативные задачи. А. А. Карацуба построил метод решения тернарных мультипликативных задач (см. [83]), который нашел в дальнейшем широкое приложение в различных областях аналитической теории чисел. Он применил свой метод к задаче о распределении произведений сдвинутых простых чисел в арифметической прогрессии, т.е. о распределении чисел вида $p\left(p^{\prime}+a\right)$, где $p, p^{\prime}$ - простые числа в арифметических прогрессиях с растущей разностью $D$, и о распределении чисел вида $\left(p^{n}+a\right) f\left(p^{\prime}\right)$, где $f-$ многочлен с целыми коэффициентами.

Пусть, как и раньше, $\omega \in(0,1 / 4], n$ - достаточно большое положительное число, $D$ простое число, $D \leqslant n^{\varkappa_{0}}$, где

$$
\varkappa_{0}=\frac{1}{4.6+\omega}, \quad(a, D)=(l, D)=1, \quad \alpha \in\left[\left(\frac{1}{2}+\omega\right) \frac{\ln D}{\ln n}, 1-4.1 \frac{\ln D}{\ln n}\right],
$$


$n_{1} \geqslant n^{1-\alpha}, n_{2} \geqslant n^{\alpha}, p, p^{\prime}-$ простые числа,

$$
\pi(x)=\sum_{p \leqslant x} 1, \quad \pi_{2}=\pi_{2}\left(n_{1}, n_{2}, a, l\right)=\sum_{\substack{p\left(p^{\prime}+a\right) \equiv l(\bmod D) \\ p \leqslant n_{1}, p^{\prime} \leqslant n_{2}}} 1 .
$$

А. А. Карацуба доказал, что существует абсолютная постоянная $\gamma>0$ такая, что

$$
\pi_{2}=\frac{1}{\phi(D)} \pi\left(n_{1}\right) \pi\left(n_{2}\right)+O\left(\left(n_{1} n_{2}\right)^{1+\varepsilon} D^{-1-\gamma \omega^{2}}\right),
$$

где постоянная в $O$ зависит только от $\omega$.

5.4. Нижние оценки сумм характеров от многочленов. А. А. Карацуба построил (см. [84]) бесконечную последовательность простых чисел $p$ и последовательность многочленов $f(x)$ вида $a x^{n}+b,(a, p)=(b, p)=1$, таких, что $f(x)$ не является квадратом многочлена по модулю $p$,

$$
\frac{4(p-1)}{\ln p} \leqslant n \leqslant \frac{8(p-1)}{\ln p}
$$

и

$$
\sum_{x=1}^{p}\left(\frac{f(x)}{p}\right)=p
$$

Иными словами, при любом $x$ значение $f(x)$ оказывается квадратичным вычетом по модулю $p$. Этот результат показывает, что оценку А. Вейля

$$
\left|\sum_{x=1}^{p}\left(\frac{f(x)}{p}\right)\right| \leqslant(n-1) \sqrt{p}
$$

вообще говоря, нельзя слишком сильно улучшить, заменив правую часть неравенства, скажем, величиной $C \sqrt{n} \sqrt{p}$, где $C$ - абсолютная постоянная.

5.5. Оценки сумм характеров от многочленов с простым аргументом. А. А. Карацубе принадлежит (см. [85]) ряд оценок сумм характеров Дирихле от многочленов второй степени для случая, когда аргумент многочлена пробегает короткую последовательность подряд идущих простых чисел. Пусть, например, $q$ - достаточно большое простое число, $p$ простые числа, $f(x)=(x-a)(x-b)$, где $a$ и $b$ - целые числа, удовлетворяющие условию $a b(a-b) \not \equiv 0(\bmod q)$, и пусть $\left(\frac{n}{q}\right)-$ символ Лежандра. Пусть $\varepsilon-$ произвольное число из интервала $(0,1 / 2)$. Тогда при любом $N>q^{3 / 4+\varepsilon}$ для суммы $S_{N}$,

$$
S_{N}=\sum_{p \leqslant N}\left(\frac{f(p)}{q}\right)
$$

справедлива оценка:

$$
\left|S_{N}\right| \ll \pi(N) q^{-\varepsilon^{2} / 100},
$$

где постоянная в знаке « зависит лишь от $\varepsilon$.

Подобная оценка была получена А. А. Карацубой и для случая, когда $p$ пробегает последовательность простых чисел, принадлежащих арифметической прогрессии, разность которой может расти вместе с модулем $q$.

А. А. Карацубой высказана гипотеза, согласно которой нетривиальная оценка суммы $S_{N}$ при $N$, "маленьких" по сравнению с $q$, остается справедливой, если заменить $f(x)$ произвольным многочленом $n$-й степени, который не является квадратом по модулю q. Эта гипотеза в настоящее время не доказана. 
5.6. Распределение пар квадратичных вычетов и невычетов вида $\boldsymbol{p}+\boldsymbol{k}$. А. А. Карацуба получил закон распределения пар квадратичных вычетов и невычетов в последовательностях вида $p+k, k \not \equiv 0(\bmod q)$, где $q$ - достаточно большое простое число.

Пусть $\tau_{1}= \pm 1, \tau_{2}= \pm 1, a, b$ - целые числа такие, что $a b(a-b) \not \equiv 0(\bmod q), \omega-$ вещественное число с условием $0<\omega<1 / 2, N>q^{3 / 4+\omega}$.

А. А. Карацуба доказал (см. [85], [86]), что в этом случае количество простых чисел $p$, не превосходящих $N$ и таких, что

$$
\left(\frac{p+a}{q}\right)=\tau_{1}, \quad\left(\frac{p+b}{q}\right)=\tau_{2},
$$

равно

$$
\frac{1}{4} \pi(N)+O\left(N q^{-0.01 \omega^{2}}\right)
$$

где постоянная в знаке $O$ зависит только от $\omega$.

5.7. Распределение степенных вычетов и первообразных корней в редких последовательностях. В 2000 г. А. А. Карацубой получены (см. [87]) нетривиальные оценки сумм значений характеров Дирихле "с весами", т.е. сумм слагаемых вида $\chi(n) f(n)$, где $f(n)-$ функция натурального аргумента. Оценки такого рода находят применение при решении широкого круга задач теории чисел, связанных с распределением степенных вычетов (невычетов), а также первообразных корней в тех или иных последовательностях.

Пусть $\chi$ - неглавный характер по модулю $q, q-$ простое число, $q \geqslant q_{1}(\delta)$, где $\delta$ - произвольное положительное число, не превосходящее $0.01, \tau_{k}(n)$ - число решений в натуральных числах $x_{1}, \ldots, x_{k}$ уравнения $x_{1} \cdots x_{k}=n, a$ и $b$ - целые числа, $(a, q)=1, a \neq \equiv(\bmod q)$.

Пусть $k \geqslant 2$ - целое число, $N \geqslant q^{1 / 2-1 /(2(k+1))+\delta}$, где $0<\delta<\min \{0.01,2 /(3(k+1))\}$, $0<|a| \leqslant \sqrt{q}$, и пусть, наконец,

$$
T_{k}(x)=\sum_{n \leqslant x} \tau_{k}(n)
$$

(асимптотическое выражение для $T_{k}(x)$ см. в. п. 4.7). Для сумм $V_{1}(x)$ и $V_{2}(x)$ величин $\tau_{k}(n)$, распространенных на значения $n \leqslant x$, для которых числа $n+a$ являются квадратичными вычетами (соответственно, невычетами) по модулю $q$, А. А. Карацуба получил асимптотические формулы вида

$$
V_{1}(x)=\frac{1}{2} T_{k}(x)+O\left(x q^{-0.01 \delta^{2}}\right), \quad V_{2}(x)=\frac{1}{2} T_{k}(x)+O\left(x q^{-0.01 \delta^{2}}\right) .
$$

Аналогично, для суммы $V(x)$ величин $\tau_{k}(n)$, взятых по всем $n \leqslant x$, для которых $n+a$ будет первообразным корнем по модулю $q$, получается асимптотическое выражение вида

$$
V(x)=\left(1-\frac{1}{p_{1}}\right) \cdots\left(1-\frac{1}{p_{s}}\right) T_{k}(x)+O\left(x q^{-0.01 \delta^{2}}\right),
$$

где $p_{1}, \ldots, p_{s}-$ все простые делители числа $q-1$.

Метод, развитый А. А. Карацубой, был применен им и к задачам о распределении степенных вычетов (невычетов) в последовательностях сдвинутых простых чисел $p+a$, чисел вида $x^{2}+y^{2}+a$ и т.д.

5.8. Суммы характеров на аддитивных последовательностях. А. А. Карацубой найден новый метод (см. [88], [89], а также [90]), позволяющий находить весьма точные оценки сумм значений неглавных характеров Дирихле на аддитивных последовательностях, т.е. на последовательностях, состоящих из чисел вида $x+y$, где переменные $x$ и $y$ независимо друг от друга пробегают соответственно некоторые множества $A$ и $B$. 
Наиболее ярким примером результатов такого рода является следующее утверждение, находящее применение при решении широкого класса задач, связанных с суммированием значений характеров Дирихле. Пусть $\varepsilon-$ произвольное положительное число, $0<\varepsilon<1 / 2, q-$ достаточно большое простое число, $\chi$ - неглавный характер по модулю $q$. Пусть, далее, $A$ и $B$ - произвольные множества натуральных чисел, не превосходящих $q$, причем $\|A\|>q^{\varepsilon}$, $\|B\|>q^{1 / 2+\varepsilon}$, где $\|A\|$ и $\|B\|$ - количества чисел множеств $A$ и $B$ соответственно. Тогда имеет место оценка

$$
\left|\sum_{x \in A} \sum_{y \in B} \chi(x+y)\right| \leqslant c\|A\| \cdot\|B\| q^{-0.05 \varepsilon^{2}}, \quad c=c(\varepsilon)>0 .
$$

Метод А.А. Карацубы позволяет получать нетривиальные оценки сумм такого рода и в некоторых случаях, когда указанные выше условия на множества $A$ и $B$ заменяются иными, например, $\|A\|>q^{\varepsilon}, \sqrt{\|A\|}\|B\|>q^{1 / 2+\varepsilon}$.

В случае же, когда $A$ и $B$ представляют собой множества простых чисел отрезков $(1, X]$, $(1, Y]$ соответственно, причем $X \geqslant q^{1 / 4+\varepsilon}, Y \geqslant q^{1 / 4+\varepsilon}$, имеет место оценка вида

$$
\left|\sum_{p \leqslant X} \sum_{p^{\prime} \leqslant Y} \chi\left(p+p^{\prime}\right)\right| \leqslant c \pi(X) \pi(Y) q^{-c_{1} \varepsilon^{2}},
$$

где $c_{1}>0$ - абсолютная постоянная, а постоянная $c$ зависит только от $\varepsilon$.

\section{6. Работы последних лет}

6.1. Теорема АТС. Работы по физике. А. А. Карацуба доказал теорему АТС (annроксимация тригонометрических сумм более короткими) общего вида (см. [91]), что позволило применять ее в задачах математики и математической физики самого широкого спектра.

Проблема приближения тригонометрического ряда какой-либо подходящей функцией рассматривалась еще Эйлером и Пуассоном. Суммы вида

$$
S=\sum_{a<k \leqslant b} \varphi(k) e^{2 \pi i f(k)},
$$

где $\varphi(x)$ и $f(x)$ - вещественные функции вещественного аргумента, появляются, например, в теории чисел при анализе дзета-функции Римана, при решении задач, связанных с распределением целых точек в различных областях на плоскости и в пространстве, при изучении рядов Фурье, при решении таких дифференциальных уравнений, как волновое уравнение, уравнение теплопроводности и т.д.

Длиной суммы $S$ называют число $b-a$ (для целых $a$ и $b$ это просто число слагаемых в $S$ ). При определенных условиях на $\varphi(x)$ и $f(x)$ сумму $S$ можно заменить с хорошей точностью другой суммой

$$
S_{1}=\sum_{\alpha<k \leqslant \beta} \Phi(k) e^{2 \pi i F(k)},
$$

длина которой $\beta-\alpha$ много меньше, чем $b-a$.

Первые соотношения вида $S=S_{1}+R$, где $R$ - остаточный член, с конкретными функциями $\varphi(x)$ и $f(x)$ были получены Г. Х. Харди и Дж. И. Литтлвудом (см. [55]), а также И. М. Виноградовым (см. [92]) и И.Г. Ван дер Корпутом (см. [93]). В каждой из работ на функции $\varphi(x)$ и $f(x)$ накладывались некоторые ограничения. С ограничениями, наиболее удобными для приложений, теорема была доказана А. А. Карацубой в [91] (см. также [7], [9], [94]).

Tеорема АTC (А.А. Карацуба). Пусть вещественные функиии $f(x)$ и $\varphi(x)$ удовлетворяют на отрезке $[a, b]$ следующим условиям:

- $f^{\prime \prime \prime \prime}(x)$ и $\varphi^{\prime \prime}(x)$ являются непрерывными; 
- существуют числа $H, U$ u $V$ такие, что $H>0,1 \ll U \ll V, 0<b-a \leqslant V$,

$$
\begin{array}{rlrl}
\frac{1}{U} \ll f^{\prime \prime}(x) & \ll \frac{1}{U}, \quad f^{\prime \prime \prime}(x) \ll \frac{1}{U V}, & f^{\prime \prime \prime \prime}(x) \ll \frac{1}{U V^{2}}, \\
\varphi(x) & \ll H, \quad \varphi^{\prime}(x) \ll \frac{H}{V}, \quad \varphi^{\prime \prime}(x) \ll \frac{H}{V^{2}} .
\end{array}
$$

Тогда, определяя числа $x_{\mu}$ из уравнения

$$
f^{\prime}\left(x_{\mu}\right)=\mu,
$$

имеем

$$
\sum_{a<\mu \leqslant b} \varphi(\mu) e^{2 \pi i f(\mu)}=\sum_{f^{\prime}(a) \leqslant \mu \leqslant f^{\prime}(b)} C(\mu) Z(\mu)+R
$$

где

$$
\begin{aligned}
& R=O\left(\frac{H U}{b-a}+H T_{a}+H T_{b}+H \log \left(f^{\prime}(b)-f^{\prime}(a)+2\right)\right), \\
& T_{j}=\left\{\begin{array}{ll}
0, & \text { если } f^{\prime}(j) \text { целое, } \\
\min \left(\frac{1}{\left\|f^{\prime}(j)\right\|}, \sqrt{U}\right), & \text { если }\left\|f^{\prime}(j)\right\| \neq 0,
\end{array} \quad j=a, b,\right. \\
& C(\mu)=\left\{\begin{array}{l}
1, \quad \text { если } f^{\prime}(a)<\mu<f^{\prime}(b), \\
\frac{1}{2}, \quad \text { если } \mu=f^{\prime}(a) \text { или } \mu=f^{\prime}(b),
\end{array}\right. \\
& Z(\mu)=\frac{1+i}{\sqrt{2}} \frac{\varphi\left(x_{\mu}\right)}{\sqrt{f^{\prime \prime}\left(x_{\mu}\right)}} e^{2 \pi i\left(f\left(x_{\mu}\right)-\mu x_{\mu}\right)} .
\end{aligned}
$$

В последние годы помимо исследований в области теории чисел А. А. Карацуба занимался некоторыми проблемами теоретической физики, в том числе в области квантовой теории поля. Путем применения своей теоремы АТС и некоторых других теоретико-числовых подходов ему удалось получить новые результаты (см. [95], [96]) по проблеме Джейнса-Каммингса в квантовой оптике.

6.2. Эффект Карацубы. В 2007-2008 гг. А. А. Карацуба установил новое свойство чисел натурального ряда, отражающее взаимозависимость их структуры и вида простых сомножителей, составляющих эти числа. Он обнаружил следующий интересный эффект: разность мощностей множеств чисел с нечетным и четным количеством простых сомножителей в случае, когда на эти сомножители накладываются некоторые ограничения, стремится к бесконечности.

Пусть $\mathbb{N}$ есть множество натуральных чисел. Пусть $\mathbb{N}_{0}$ и $\mathbb{N}_{1}$ - подмножества $\mathbb{N}$ такие, что $n \in \mathbb{N}_{0}$, если $n$ содержит четное число простых сомножителей, и $n \in \mathbb{N}_{1}$, если $n$ содержит нечетное число простых сомножителей. Обозначим через $n_{0}(x)$ и $n_{1}(x), x \geqslant 1$, количество чисел $n$ из $\mathbb{N}_{0}$ и из $\mathbb{N}_{1}$ соответственно и таких, что $n \leqslant x$. Через $n(x)$ обозначим количество чисел $n$, не превосходящих $x$, все простые делители которых принадлежат заданным арифметическим прогрессиям с разностью $k, k \geqslant 2$, и первыми членами $l_{1}, l_{2}, \ldots, l_{r}$, где $1 \leqslant l_{1}<\cdots<l_{r}<k, r \leqslant \varphi(k), \varphi(k)-$ функция Эйлера, $\left(l_{1}, k\right)=1, \ldots,\left(l_{r}, k\right)=1$.

Э. Ландау (см. [97]) доказал, что

$$
n_{0}(x) \sim n_{1}(x) \sim \frac{1}{2} x
$$

причем разница между $n_{0}(x)$ и $n_{1}(x)$ мала:

$$
n_{1}(x)-n_{0}(x)=O\left(x e^{-c \sqrt{\ln x}}\right),
$$


и

$$
n(x)=c_{0} x(\ln x)^{r / \varphi(k)-1}+O\left(x(\ln x)^{r / \varphi(k)-2}\right) .
$$

Аналогичные результаты, но со специальными множествами простых чисел, отличными от множеств Ландау, были получены учеником А. А. Карацубы М. Е. Чангой (см. [98]).

Обозначим через $\mathbb{A}$ множество простых чисел, принадлежащих прогрессиям $k m+l_{j}, j \leqslant r$. Исключим из рассмотрения тривиальные случаи $r=0$ и $r=\varphi(k)$ и будем считать в дальнейшем, что $1 \leqslant r<\varphi(k)$.

В 2007-2008 гг. А. А. Карацуба нашел следующее свойство чисел натурального ряда.

Теорема (А. А. Карацуба). Пусть $\mathbb{N}^{*}$ - множество натуралъных чисел $n$, не имеющих простых делителей из $\mathbb{A}, \mathbb{N}_{0}^{*} \subset \mathbb{N}^{*}$ содержит только числа $n$ с четным числом простых делителей, $\mathbb{N}_{1}^{*} \subset \mathbb{N}^{*}$ содержит только числа $n$ с нечетным числом простых делителей. Пусть

$$
n^{*}(x)=\sum_{\substack{n \leqslant x \\ n \in \mathbb{N}^{*}}} 1, \quad n_{0}^{*}(x)=\sum_{\substack{n \leqslant x \\ n \in \mathbb{N}_{0}^{*}}} 1, \quad n_{1}^{*}(x)=\sum_{\substack{n \leqslant x \\ n \in \mathbb{N}_{1}^{*}}} 1 .
$$

Имеет место асимптотическая формула

$$
n_{1}^{*}(x)-n_{0}^{*}(x) \sim C n^{*}(x)(\ln x)^{2(r / \varphi(k)-1)}, \quad x \rightarrow+\infty .
$$

Эта и другие теоремы сформулированы и доказаны в [99], [100] на основе рукописных черновиков А.А. Карацубы. Проиллюстрировать эффект Карацубъ можно на следующих примерах (см. [99], [100]).

Рассмотрим натуральные числа, в каноническое представление которых не входят простые числа, принадлежащие арифметической прогрессии $6 m+1, m=1,2, \ldots$. Тогда

$$
n_{1}^{*}(x)-n_{0}^{*}(x) \sim \frac{\pi}{8 \sqrt{3}} \frac{n^{*}(x)}{\ln x}, \quad x \rightarrow+\infty .
$$

Пусть $\widetilde{\mathbb{N}}$ - множество натуральных чисел, представимых суммой двух квадратов целых чисел, $\widetilde{\mathbb{N}}_{0}-$ подмножество $\widetilde{\mathbb{N}}$, включающее числа с четным количеством простых сомножителей, $\widetilde{\mathbb{N}}_{1}$ - подмножество $\widetilde{\mathbb{N}}$, состоящее из чисел с нечетным количеством простых сомножителей. Как и выше, определим следующие функции:

$$
\widetilde{n}(x)=\sum_{\substack{n \leqslant x \\ n \in \mathbb{N}}} 1, \quad \widetilde{n}_{0}(x)=\sum_{\substack{n \leqslant x \\ n \in \widetilde{\mathbb{N}}_{0}}} 1, \quad \widetilde{n}_{1}(x)=\sum_{\substack{n \leqslant x \\ n \in \widetilde{\mathbb{N}}_{1}}} 1 .
$$

Тогда

$$
\widetilde{n}_{1}(x)-\widetilde{n}_{0}(x) \sim \frac{\pi}{6} \frac{\widetilde{n}(x)}{\ln x}, \quad x \rightarrow+\infty
$$

\section{Список литературы}

[1] А.А. Карацуба, "Решение одной задачи из теории конечных автоматов", УМН, 15:3 (1960), $157-159$.

[2] А. А. Карацуба, Ю. П. Офман, "Умножение многозначных чисел на автоматах", ДАН $C C C P$, 145:2 (1962), 293-294.

[3] J.-P. Delahaye, "Mathematiques et philosophie", Pour la Science, 277 (2000), 100-104.

[4] А. А. Карацуба, Список научных публикащий, http://www.mi.ras.ru/ karatsuba/list.html.

[5] А. А. Карацуба, Основы аналитической теории чисел, Наука, М., 1975. 
[6] Г. И. Архипов, А.А. Карацуба, В.Н. Чубариков, Теория кратных тригонометрических сумм, Наука, М., 1987.

[7] С. М. Воронин, А. А. Карацуба, Дзета-функиия Римана, Физматлит, М., 1994.

[8] A. A. Karatsuba, Complex Analysis in Number Theory, CRC Press, Boca Raton, FL, 1995.

[9] G. I. Arkhipov, V. N. Chubarikov, A. A. Karatsuba, Trigonometric Sums in Number Theory and Analysis, de Gruyter Exp. Math., 39, Walter de Gruyter, Berlin, 2004.

[10] Г. И. Архипов, В.Н.Чубариков, "О математических работах профессора А. А. Карацубы", Аналитическая теория чисел и приложения, Сборник статей. K 60-летию со дня рождения профессора Анатолия Алексеевича Карацубы, Тр. МИАН, 218, Наука, М., 1997, 7-19.

[11] E. F. Moore, "Gedanken-experiments on sequential machines", Automata Studies, Ann. of Math. Stud., 34, Princeton Univ. Press, Princeton, NJ, 1956, 129-153.

[12] A. Karacuba, "Berechnungen und die Kompliziertheit von Beziehungen", Elektron. Inform.-verarb. Kybernetik, 11 (1975), 603-606.

[13] А. А. Карацуба, "Сложность вычислений”, Оптимальное управление и дифференииальные уравнения, Сборник статей. К семидесятилетию со дня рождения академика Евгения Фроловича Мищенко, Тр. МИАН, 211, Наука, Физматлит, М., 1995, 186-202.

[14] Д. Кнут, Искусство программирования. Т. 2. Получисленные алгоритмы, Мир, М., 2000.

[15] A. Schönhage, V. Strassen, "Schnelle Multiplikation großer Zahlen", Computing, 7:3-4 (1971), 281-292.

[16] V. Strassen, "Gaussian elimination is not optimal", Numer. Math., 13:4 (1969), 354-356.

[17] А.А. Карацуба, "Оценки тригонометрических сумм особого вида и их приложения", ДАН CCCP, 137:3 (1961), 513-514.

[18] А. А. Карацуба, "Аналог проблемы Варинга", Вестн. Моск. ун-та. Сер. 1. Матем., мех., 1962, № $1,38-46$.

[19] А. А. Карацуба, "Распределение дробных долей многочленов специального вида", Вестн. Моск. ун-та. Сер. 1. Матем., мех., 1962, № 3, 34-39.

[20] А. А. Карацуба, "Проблема Варинга для сравнения по модулю, равному степени простого числа", Вестн. Моск. ун-та. Сер. 1. Матем., мех., 1962, № 4, 28-38.

[21] А. А. Карацуба, Н. М. Коробов, "О теореме о среднем", ДАН СССР, 149:2 (1963), 245-248.

[22] А.А. Карацуба, "Тригонометрические суммы специального вида и их приложения", Изв. АH СССР. Сер. матем., 28:1 (1964), 237-248.

[23] А. А. Карацуба, “Об оценке числа решений некоторых уравнений”, ДАН СССР, 165:1 (1965), 31-32.

[24] А. А. Карацуба, “О системах сравнений”, Изв. АН СССР. Сер. матем., 29:4 (1965), 935-944.

[25] А.А. Карацуба, “Теоремы о среднем и полные тригонометрические суммы”, Изв. АН CCCP. Сер. матем., 30:1 (1966), 183-206.

[26] А.А. Карацуба, Метод тригонометрических сумм и теоремы о среднем, Дис. ... докт. физ.-матем. наук, МИАН, М., 1966.

[27] А. А. Карацуба, "Метод тригонометрических сумм и теоремы о среднем”, Матем. заметки, 1:1 (1967), 99-110.

[28] А.А. Карацуба, "Среднее значение модуля тригонометрической суммы”, Изв. АН СССР. Сер. матем., 37:6 (1973), 1203-1227.

[29] Г. И. Архипов, А. А. Карацуба, В. Н. Чубариков, "Верхняя граница модуля кратной тригонометрической суммы", Аналитическал теория чисел, математический анализ и их приложения, Сборник статей. Посвящается академику Ивану Матвеевичу Виноградову к его восьмидесятипятилетию, Тр. МИАН СССР, 143, 1977, 3-31.

[30] Г. И. Архипов, А.А. Карацуба, В.Н. Чубариков, "Кратные тригонометрические суммы", Тр. МИАН СССР, 151, 1980, 3-128.

[31] Г.И. Архипов, А.А. Карацуба, В.Н. Чубариков, "Кратные тригонометрические суммы и их приложения", Изв. АН СССР. Сер. матем., 44:4 (1980), 723-781.

[32] Г. И. Архипов, А. А. Карацуба, В.Н. Чубариков, "Особые случаи теории кратных тригонометрических сумм", Изв. АН СССР. Сер. матем., 47:4 (1983), 707-784. 
[33] И. М. Виноградов, А. А. Карацуба, "Метод тригонометрических сумм в теории чисел", Алгебра, математическая логика, теория чисел, топология, Сборник обзорных статей. 1. K 50-летию Института, Тр. МИАН СССР, 168, 1984, 4-30.

[34] U. V. Linnik, "On Weyl's sums", Матем. сб., 12:1 (1943), 28-39.

[35] Г. И. Архипов, “Теорема о среднем значении модуля кратной тригонометрической суммы”, $M a$ тем. заметки, 17:1 (1975), 143-153.

[36] Г. И. Архипов, А.А. Карацуба, В.Н. Чубариков, “Тригонометрические интегралы", Изв. АН СССР. Сер. матем., 43:5 (1979), 971-1003.

[37] И. М. Виноградов, Избранные труды, Изд-во АН СССР, М., 1952.

[38] М. А. Чахкиев, “О показателе сходимости особого интеграла многомерного аналога проблемы Терри”, Изв. РАН. Сер. матем., 67:2 (2003), 211-224.

[39] А. А. Карацуба, "О функции $G(n)$ в проблеме Варинга", Изв. АН СССР. Сер. матем., 49:5 (1985), 935-947.

[40] Г. И. Архипов, А.А. Карацуба, “О локальном представлении нуля формой”, Изв. АН CCCP. Сер. матем., 45:5 (1981), 948-961.

[41] А. А. Карацуба, "Распределение обратных величин в кольце вычетов по заданному модулю", ДАН, 333:2 (1993), 138-139.

[42] А.А. Карацуба, “Дробные доли специального вида функций”, Изв. РАН. Сер. матем., 59:4 (1995), 61-80.

[43] А. А. Карацуба, “Аналоги сумм Клоостермана", Изв. РАН. Сер. матем., 59:5 (1995), 93-102.

[44] А.А. Карацуба, "Аналоги неполных сумм Клоостермана и их приложения”, Number Theory, Tatra Mt. Math. Publ., 11, 1997, 89-120.

[45] А. А. Карацуба, “Двойные суммы Клоостермана”, Матем. заметки, 66:5 (1999), 682-687.

[46] J. Friedlander, H. Iwaniec, "The Brun-Titchmarsh theorem", Analytic Number Theory, London Math. Soc. Lecture Note Ser., 247, Cambridge Univ. Press, Cambridge, 1997, 85-93.

[47] J. Friedlander, H. Iwaniec, "The polynomial $X^{2}+Y^{4}$ captures its primes", Ann. of Math. (2), 148:3 (1998), 945-1040.

[48] D. R. Heath-Brown, "The largest prime factor of $X^{3}+2$ ", Proc. London Math. Soc. (3), 82:3 (2001), 554-596.

[49] А.А. Глибичук, "Комбинаторные свойства множеств вычетов по простому модулю и задача Эрдёша-Грэхэма", Матем. заметки, 79:3 (2006), 384-395.

[50] А. А. Карацуба, "О нулях функции $\zeta(s)$ на коротких промежутках критической прямой”, Изв. АН СССР. Сер. матем., 48:3 (1984), 569-584.

[51] А. А. Карацуба, "Распределение нулей функции $\zeta(1 / 2+i t)$ ", Изв. АН СССР. Сер. матем., 48:6 (1984), 1214-1224.

[52] А. А. Карацуба, “О нулях дзета-функции Римана на критической прямой”, Современные проблемы математики. Математический анализ, алгебра, топология, Сборник статей. Посвящается академику Льву Семеновичу Понтрягину к его семидесятипятилетию, Тр. МИАН СССР, 167, 1985, 167-178.

[53] A. Selberg, "On the zeros of Riemann's zeta function", Skr. Norske Vid. Akad. Oslo I, 10 (1942), $1-59$.

[54] А. А. Карацуба, "О количестве нулей дзета-функции Римана, лежащих на почти всех коротких промежутках критической прямой”, Изв. РАН. Сер. матем., 56:2 (1992), 372-397.

[55] G.H. Hardy, J.E. Littlewood, "Contributions to the theory of the Riemann zeta-function and the theory of the distribution of primes", Acta Math., 41:1 (1916), 119-196.

[56] Я. Мозер, “Об одной сумме в теории дзета-функции Римана”, Acta Arith., 31:1 (1976), 31-43; "Об одной теореме Харди-Литтлвуда в теории дзета-функции Римана", Acta Arith., 31:1 (1976), 45-51; "Добавление к работе: "Об одной теореме Харди-Литтлвуда в теории дзета-функции Римана", Acta Arith., 35:4 (1979), 403-404; "Исправление к работам: Acta Arith. 31 (1976), стр. 31-43; 31 (1976), стр. 45-51; 35 (1979), стр. 403-404", Acta Arith., 40:1 (1981), 97-107. 
[57] А.А. Карацуба, "О расстоянии между соседними нулями дзета-функции Римана, лежащими на критической прямой”, Теория чисел, математический анализ и их приложения, Сборник статей. Посвящается академику Ивану Матвеевичу Виноградову к его девяностолетию, Тр. МИАН СССР, 157, 1981, 49-63.

[58] А. А. Карацуба, “Дзета-функция Римана и ее нули”, УМН, 40:5 (1985), 19-70.

[59] А. А. Лаврик, "Равномерные приближения и нули в коротких интервалах производных $Z$-функции Харди", Anal. Math., 17:4 (1991), 257-279.

[60] A. Selberg, "Contributions to the theory of the Riemann zeta-function", Arch. Math. Naturvid., 48:5 (1946), 89-155.

[61] А. А. Карацуба, "Плотностная теорема и поведение аргумента дзета-функции Римана", Матем. заметки, 60:3 (1996), 448-449.

[62] А. А. Карацуба, "О функции $S(t)$ ", Изв. РАН. Сер. матем., 60:5 (1996), 27-56.

[63] А. А. Карацуба, "Омега-теоремы для дзетовых сумм", Матем. заметки, 73:2 (2003), 228-233.

[64] А. А. Карацуба, "О нулях функции Дэвенпорта-Хейльбронна, лежащих на критической прямой”, Изв. АН СССР. Сер. матем., 54:2 (1990), 303-315.

[65] H. Davenport, H. Heilbronn, "On the zeros of certain Dirichlet series", J. London Math. Soc., 11:3 (1936), 181-185; "On the zeros of certain Dirichlet series. II", J. London Math. Soc., 11:4 (1936), 307-312.

[66] С. М. Воронин, "О нулях некоторых рядов Дирихле, лежащих на критической прямой”, Изв. АН СССР. Сер. матем., 44:1 (1980), 63-91.

[67] A.A. Karatsuba, "On zeros of the Davenport-Heilbronn function", Proceedings of the Amalfi Conference on Analytic Number Theory (Maiori, 1989), Univ. Salerno, Salerno, 1992, 271-293.

[68] А.А. Карацуба, "Уточнение теорем о количестве нулей, лежащих на отрезках критической прямой, некоторых рядов Дирихле", УМН, 47:2 (1992), 193-194.

[69] А. А. Карацуба, "О нулях специального вида функций, связанных с рядами Дирихле”, Изв. АH СССР. Сер. матем., 55:3 (1991), 483-514.

[70] А. А. Карацуба, "Равномерная оценка остаточного члена в проблеме делителей Дирихле", Изв. АН СССР. Сер. матем., 36:3 (1972), 475-483.

[71] Е.К. Титчмарш, Теория дзета-функиии Римана, ИЛ, М., 1953.

[72] H.-E. Richert, "Einführung in die Theorie der starken Rieszschen Summierbarkeit von Dirichletreihen", Nachr. Akad. Wiss. Göttingen Math.-Phys. Kl. II, 1960, 17-75.

[73] A. A. Karatsuba, "The multidimensional Dirichlet divisor problem and zero free regions for the Riemann zeta function", Funct. Approx. Comment. Math., 28 (2000), 131-140.

[74] А. А. Карацуба, "О связи многомерной проблемы делителей Дирихле с границей нулей $\zeta(s)$ ", Матем. заметки, 70:3 (2001), 477-480.

[75] А.А. Карацуба, "О нижних оценках максимума модуля $\zeta(s)$ в малых областях критической полосы", Матем. заметки, 70:5 (2001), 796-797.

[76] А. А. Карацуба, "О нижних оценках максимума модуля дзета-функции Римана на коротких промежутках критической прямой”, Изв. РАН. Сер. матем., 68:6 (2004), 99-104.

[77] R. Balasubramanian, "On the frequency of Titchmarsh's phenomenon for $\zeta(s)$. IV", Hardy-Ramanujan J., 9 (1986), 1-10.

[78] А.А. Карацуба, "Суммы характеров и первообразные корни в конечных полях", ДАН СССР, 180:6 (1968), 1287-1289.

[79] А. А. Карацуба, "Об оценках сумм характеров", Изв. АН СССР. Сер. матем., 34:1 (1970), 20-30.

[80] А. А. Карацуба, “Суммы характеров с простыми числами”, Изв. АН СССР. Сер. матем., 34:2 (1970), 299-321.

[81] И. М. Виноградов, “Оценка одной суммы, распространенной на простые числа арифметической прогрессии", Изв. АН СССР. Сер. матем., 30:3 (1966), 481-496.

[82] А.А. Карацуба, "Суммы характеров по последовательности сдвинутых простых чисел и их применения", Матем. заметки, 17:1 (1975), 155-159.

[83] А.А. Карацуба, "Распределение произведений сдвинутых простых чисел в арифметических прогрессиях", ДАН СССР, 192:4 (1970), 724-727. 
[84] А.А. Карацуба, "Об оценках снизу сумм характеров от многочленов", Матем. заметки, 14:1 (1973), 67-72.

[85] А. А. Карацуба, “Суммы символов Лежандра от многочленов второй степени с простыми числами", Изв. АН СССР. Сер. матем., 42:2 (1978), 315-324.

[86] А. А. Карацуба, "О распределении значений неглавных характеров", Теория чисел, математический анализ и их приложения, Сборник статей. Посвящается академику Ивану Матвеевичу Виноградову к его восьмидесятипятилетию, Тр. МИАН СССР, 142, 1976, 156-164.

[87] А. А. Карацуба, “Суммы характеров с весами”, Изв. РАН. Сер. матем., 64:2 (2000), $29-42$.

[88] А.А. Карацуба, "Распределение значений характеров Дирихле на аддитивных последовательностях", ДАН СССР, 319:3 (1991), 543-545.

[89] A.A. Karatsuba, "Sums of characters with prime numbers and their applications", Number Theory, Tatra Mt. Math. Publ., 20, 2000, 155-162.

[90] А. А. Карацуба, “Арифметические проблемы теории характеров Дирихле”, УМН, 63:4(382) (2008), 43-92.

[91] A. A. Karatsuba, "Approximation of exponential sums by shorter ones", Proc. Indian Acad. Sci. Math. Sci., 97:1-3 (1987), 167-178.

[92] И. М. Виноградов, "О среднем значении числа классов чисто коренных форм отрицательного определителя", Сообщ. Харъковск. матем. о-ва, 16:1-2 (1918), 10-38.

[93] J. G. van der Corput, "Verschärfung der Abschätzung beim Teilerproblem", Math. Ann., 87:1-2 (1922), 39-65.

[94] А.А. Карацуба, М.А. Королёв, “Теорема о приближении тригонометрической суммы более короткой”, Изв. РАН. Сер. матем., 71:2 (2007), 123-150.

[95] A. A. Karatsuba, E. A. Karatsuba, "Applications of ATS in a quantum-optical model", Analysis and Mathematical Physics, Trends Math., Birkhäuser Verlag, Basel, 2009, 211-232.

[96] A.A. Karatsuba, E. A. Karatsuba, "A resummation formula for collapse and revival in the Jaynes-Cummings model", J. Phys. A: Math. Theor., 42:19 (2009), 195304.

[97] E. Landau, Handbuch der Lehre von der Verteilung der Primzahlen, Zweiter Band, Teubner, Leipzig, 1909.

[98] М.Е. Чанга, "О числах, все простые делители которых лежат в специальных промежутках", Изв. РАН. Сер. матем., 67:4 (2003), 213-224.

[99] А. А. Карацуба, "Об одном свойстве множества простых чисел как мультипликативного базиса натурального ряда", ДАН, 439:2 (2011), 159-162.

[100] А. А. Карацуба, “Об одном свойстве множества простых чисел”, УМН, 66:2 (2011), 3-14. 